Old Dominion University

ODU Digital Commons

Bioelectrics Publications

Frank Reidy Research Center for Bioelectrics

2012

\title{
Enhanced Killing Effect of Nanosecond Pulse Electric Fields on PANC1 and Jurkat Cell Lines in the Presence of Tween 80
}

Gaurav Basu

Bhargava Subhash Kalluri

Ahmet Can Sabuncu

Old Dominion University

Christopher J. Osgood

Old Dominion University

Michael W. Stacey

Old Dominion University, mstacey@odu.edu

Follow this and additional works at: https://digitalcommons.odu.edu/bioelectrics_pubs

Part of the Biomedical Engineering and Bioengineering Commons, and the Cell and Developmental Biology Commons

\section{Repository Citation}

Basu, Gaurav; Kalluri, Bhargava Subhash; Sabuncu, Ahmet Can; Osgood, Christopher J.; and Stacey, Michael W., "Enhanced Killing Effect of Nanosecond Pulse Electric Fields on PANC1 and Jurkat Cell Lines in the Presence of Tween 80" (2012). Bioelectrics Publications. 62.

https://digitalcommons.odu.edu/bioelectrics_pubs/62

This Article is brought to you for free and open access by the Frank Reidy Research Center for Bioelectrics at ODU Digital Commons. It has been accepted for inclusion in Bioelectrics Publications by an authorized administrator of ODU Digital Commons. For more information, please contact digitalcommons@odu.edu. 


\section{Enhanced killing effect of Nanosecond Pulse Electric Fields (nsPEFs) on PANC1 and Jurkat cell lines in the presence of Tween 80}

Gaurav Basu*, Bhargava Subhash Kalluri*, Christopher J. Osgood, and Michael W. Stacey.

Frank Reidy Research Center for Bioelectrics, Old Dominion University, Norfolk, VA 23508.

*Authors equally contribute towards the manuscript.

\section{Abstract:}

We investigated the effects of nanosecond pulsed electric fields (nsPEFs) on Jurkat and PANC1 cells which are human carcinoma cell lines in the presence of Tween 80 (T80) at a concentration of $0.18 \%$ and demonstarted an enhanced killing effect of nsPEFs in the presence of T80. We used two biological assays to determine the cell viability after exposing the cells to nsPEFs in the presence of T80 and observed a significant increase in the killing effects of nsPEFs. We did not see a toxic effect of T80 when the cells were exposed to the surfactant alone. However, we saw a synergistic effect when cells exposured to T80 was combined with the nsPEFs. Increasing the time of exposure for up to 8 hours in T80 led to a significant decrease in cell viability when nsPEFs were applied to the cells compared to control cells. We also observed cell-type specific swelling in the presence of T80. We suggest that T80 acts as an adjuvant in facilitating the effects of nsPEFs on the cell membrane, however, the limitations of the viability assays will be discussed. We conclude that T80 may increase the fragility of the cell membrane which makes it more succeptible to nsPEFs mediated killing.

Keywords: Nanosecond pulse electric fields, Tween 80, PANC1 cells, Jurkat cells

Corresponding author: Michael W. Stacey, Address: Frank Reidy center for Bioelectrics, 4211 Monarch Way, STE-300, Norfolk, Virginia 23508, USA. Telephone: +1-757-6832245, Fax: +1-757-451-1010.

E-mail address: mstacey@odu.edu

\section{Introduction:}

The use of nanosecond pulse electric fields for the treatment of tumors is a relatively new and exciting field which is being explored by a number of groups around the world. The charging time constant for a typical mammalian cell (of $10 \mu \mathrm{m}$ diameter) is in the order of 100 nanoseconds (ns) (Schwan H. et al. 1985). Pulsed electric field 
exposures with durations less than 100 ns can effectively penetrate into a mammalian cell. These ultra-short electric pulses are known as nanosecond Pulse Electric Fields (nsPEFs) and have shown to induce apoptosis in HCT116 human colon carcinoma cells, Jurkat cells, B10-2 fibro sarcoma tumors (Beebe S. et al. 2003), and calcium bursts in Jurkat cells (Vernier P. et al. 2003). It has been observed that when high voltage electric pulses of short time intervals $(12 \mathrm{kV} / \mathrm{cm}, 60 \mathrm{~ns})$ were applied to Jurkat, $\mathrm{GH} 3$, and PC-12 cells, there was a significant decrease in the membrane potential and increased cell death (Pakhomov A. et al. 2007). The major advantage of nanosecond pulsed electric fields (nsPEFs) is its unique ability to non-invasively induce apoptosis without generating any huge thermal effect (Nuccitelli R. et al. 2006). Although nsPEFs significantly decrease tumor volume by $80 \%$ in mice, histological results have shown that there was a significant damage to the healthy peripheral skin due to application of high electric fields (Chen X. et al. 2009). Increase in heart and respiratory rates were also observed when nsPEFs were applied to treat melanomas in mice (Chen X. et al. 2009). Keeping these characteristics in mind, nsPEFs provide a new approach to physically target tumor cells.

Polysorbate 80 is a non-ionic surfactant which is commercially known as Tween 80 (T80) (Zheng Z. and Obbard J. 2002), which can be used as an adjuvant along with different cancer treating drugs (Tsujino I. et al. 1999). The killing effect shown by Actinomycin-D and Daunomycin on drug resistant Chinese hamster cells was directly proportional to concentration of adjuvant (T80) used (Riehm H and Biedler J. 1972). Tween 80 also enhances the chemotherapeutic efficiency of drugs such as Adriamycin (Chitnis M. et al. 1984; Parris C. et al. 1987), VP16 (Tsujino I. et al. 1999), Epodyl, 
Mitomycin-C, and Thiotepa (Parris C. et al. 1987) by altering permeability of the plasma membranes of cancer cells in vitro (Riehm $\mathrm{H}$ and Biedler J. 1972). These studies show the significance of T80 as an adjuvant in effectively killing cancer cells.

Our present work focuses on enhancing the killing efficiency of nsPEFs by (using T80 as an adjuvant) exposing PANC1 and Jurkat cells to T80. We believe that exposure of cells to T80 would compromise the integrity of plasma membrane of cells, thereby increasing the effect of nsPEFs on intracellular structures of cells (as discussed above). The use of two separate viability assays confirms increased cell killing, however, the limitations of these viability assays will be discussed.

\section{Materials \& methods:}

\section{Cells \& cell culture:}

The PANC1 and Jurkat cells used in the study were purchased from American Type Culture Collection, Manassas, VA. The PANC1 cell line (human pancreatic carcinoma cell line) were chosen as an adherent cell line, whereas Jurkat cell line (human T-lymphocyte cell line), was chosen as a non-adherent cell line. The cells were maintained at $5 \% \mathrm{CO}_{2}$ and $37^{\circ} \mathrm{C}$ in humidified cell culture incubator. Dulbecco's Modified Eagle's Media (DMEM, American Type Culture Collection, Manassas, VA) supplemented with 10\% Fetal Calf Serum, 2 mM L-glutamine and 2\% of Penicillin and Streptomycin was used for culturing PANC1cells, while Roswell Park Memorial Institute media (RPMI, American Type Culture Collection, Manassas, VA) supplemented with 10\% Fetal Calf Serum, 2mM L-glutamine and 2\% of Penicillin and Streptomycin was 
used for culturing Jurkat cells. Exponentially growing cells were used for all the experiments.

\section{Nanosecond Pulse Generator:}

A transmission line pulse generator was used to deliver electric pulses of 60 nanosecond pulse duration (as shown in fig.1) (Kolb J. et al. 2006). The pulse amplitude could be adjusted by varying the gap distance of any spark gap operated in open (atmospheric pressure) air.

\section{Figure.1}

\section{Experimental setup for exposure to ultra short electric pulses:}

An optimal amount of T80 at $0.18 \%$ was used for good dispersion which had the least toxic effect on PANC1 and Jurkat cells (Sabuncu A. et al. 2010). Cell concentration was adjusted to $1 \times 10^{6}$ per $\mathrm{ml}$. and $900 \mu \mathrm{l}$ of media with suspended cells was used for experiments. To these cells, $200 \mu \mathrm{l}$ of $1 \%$ T80 was added to bring final concentration to $0.18 \%, 400 \mu \mathrm{l}$ of sample was exposed to 12 pulses of $30 \mathrm{kV} / \mathrm{cm}$ (60 ns,

3.2 Joules) using electroporation cuvettes (BioSmith Biotech Inc., San Diego, CA). After exposure, cell viability was analyzed using Trypan Blue exclusion method (Tennant $\mathrm{J}$. 1964) and WST-1 cell viability assay (Peskin A. and Winterbourn C. 2000) immediately after exposure to nsPEFs and after 8 hours.

\section{Cell viability Assays:}

Cells exposed to nsPEFs were diluted at a concentration of 1:1 with $0.4 \%$ Trypan Blue solution (Sigma Chemicals, St. Louis, MO).Viable cells were counted using a 
hemacytometer (Hausser Scientific, Horsham, PA). The Trypan Blue assay is based on the uptake of the dye the cell membrane of the dead cells however; it is not able to differentiate between apoptotic and necrotic cells. For the WST-1 cell viability assay100 $\mu \mathrm{l}$ of each sample $\left(8 \times 10^{4}\right.$ cells $)$ was transferred into each well of 96 well plate and $10 \mu \mathrm{l}$ of WST-1 (Roche Applied Sciences, Indianapolis, IA) reagent was added to each well and incubated for 1 hour. After incubation, the 96 well plate was read using micro plate reader at $450 \mathrm{~nm}$. The WST-1 cell viability assay is based on the cleavage of watersoluble tetrazolium salt WST-1 into a soluble formazan dye by a complex cell surface mechanism of metabolically active and live cells. The optical density of the formazan dye is measured to get an estimate of live cells.

\section{Cell size measurement using microscopy:}

PANC1 and Jurkat cells were viewed under bright field microscope (Olympus, Center Valley, PA) before and post exposure (5 minutes) to nsPEFs. Electronic images were captured using a CCD camera through an Olympus BX51 and cell sizes measured and analyzed using Image J software (NIH, Bethesda, MD).

\section{Statistical analysis:}

The cell viability assays were performed in triplicate, results are shown as mean, -/+standard error of mean (SEM). Statistical analysis was performed using GraphPad Prism $^{\text {TM }}$ (GraphPad software, La Jolla, CA). Two-way ANOVA was used to demonstrate the significance among the triplicate samples and two-tailed paired $t$ test $(95 \%$ 
confidence Interval) was used to detect significant difference between any two different samples. For all statistical analysis, $\mathrm{P}<0.05$ was significant.

\section{Results:}

Cell viability of Jurkat and PANC1 cells was analysed immediately after exposure to nsPEFs and 8 hours post exposure. The cell viability after exposure was compared to cells that were not exposed to T80 or nsPEFs (control) cells and cells exposed to nsPEF only (control exposure).

\section{Figure.2}

The cell viability of Jurkat cells which were exposed to nsPEFs in presence of 0.18\% T80 was assessed by Trypan Blue exclusion method and WST-1 cell viability assay (Fig. 2). The results of Trypan Blue exclusion method (Figure 2.A) and WST-1 viability assay (Fig.2.B) immediately after exposure to nsPEFs signifies that there was no toxic effect of T80 on Jurkat cells, when compared to control cells. The Trypan Blue exclusion method results also indicate that the cell viability decreased by $42 \%$ in Jurkat cells that were exposed to nsPEFs in presence of T80 which was highly significant when compared to control exposed cells $(\mathrm{P}<0.01$, respectively). Whereas, WST-1 viability assay results of Jurkat cells (Fig.2.B) indicate that there was no significant difference in the cell viability after the exposure to T80 alone when compared to the control cells. It is also indicated that there was a significant decrease in the cell viability by $58 \%$ after exposure to nsPEFs in the presence of T80, when compared to control exposed cells $(P<0.01)$. When exposed to nsPEFs for longer periods ( 8 hours) the Jurkat cells (Fig. 2.C) demonstarted a significant effect of T80 alone on cell viability 
(without exposure to nsPEFs) $(P<0.05)$. These results also demonstrates that, the cell viability decreased significantly by $62 \%$ in Jurkat cells that were exposed to nsPEFs in presence of T80, when compared to control exposed cells $(P<0.001)$. The WST-1 cell viability assay results of Jurkat cells, 8 hours after exposure to nsPEFs (Fig. 2.D) demonstrates that there was no significant toxic effect of T80 on Jurkat cells. It can be infered from results that there was a significant decrease in the cell viability by $55 \%$ in cells were exposed to nSPEFs in the presence of T80, when compared to control exposed cells $(P<0.01)$.

\section{Figure.3}

PANC1 cells that were exposed to nsPEFs in presence of T80 were assessed just after exposure to nsPEFs and after 8 hour time period by Trypan Blue exclusion method (Fig. 3.A) and WST-1 cell viability assay (Fig. 3.B). Trypan Blue exclusion method demonstrated that the cell viability decreased significantly by $52 \%$ in PANC1 cells which were exposed to nsPEFs in presence of T80, when compared to control exposed cells $(\mathrm{P}<0.01)$. There was no significant toxic effect of T80 alone on PANC1 cells (Fig. 3.C). WST-1 cell viability assay results demonstrate that there was a significant decrease in the cell viability by $58 \%$ in cells were exposed to nsPEFs in the presence of T80 (Fig. 3.B), when compared to control exposed cells $(P<0.01)$. Analysing 8 hours after exposure to nsPEFs, Trypan Blue exclusion method results indicate that there was a highly significant decrease in the cell viability by $32 \%$ in cells were exposed to nSPEFs in the presence of T80, when compared to control exposed cells $(P<0.001)$. The WST-1 cell viability results of PANC1 (Fig. 3.D) indicate that there was no significant toxic effect of T80 alone on the viability of PANC1 cells. There was a 
very significant decrease in the cell viability by $35 \%$ in cells that were exposed to nsPEFs in the presence of T80, when compared to control exposed $(P<0.001)$.

We also measured the size of Jurkat and PANC1 cells (Fig. 4) prior and post exposure to nsPEFs (5 minutes). We observed that the size of Jurkat cells (Fig.4A) increased significantly in the presence of $0.18 \%$ T80 $(P<0.05)$. There was significant change in the size of Jurkat cells in the presence of $0.18 \%$ T80 prior or post exposure to nsPEFs $(P<0.05)$. There was no significant difference in the PANC1 cell's size post exposure to nsPEFs in the presence of $0.18 \%$ T80 or cell culture media (Fig. 4B).

\section{Figure.4}

\section{Discussion:}

Our results clearly indicate that there was no immeadiate significant toxic effect of $0.18 \%$ T 80 on the viability of Jurkat and PANC1 cells, however, after 8 hours exposure Jurkat cells showed increased sensitivity. There was a significant reduction in the cell viability of PANC1 and Jurkat cells when exposed to nSPEFs in the presence of $0.18 \%$ T80 (Fig. 2.A, 2.B \& Fig. 3.A, 3.B). The decrease in cell viability was even higher 8 hours after exposure to nsPEFs in presence of $0.18 \%$ T80 (Fig. 2.C, 2.D \& Fig. 3.C, 3.D). It has been shown in the past that $\mathrm{T} 80$ has similar adjuvant activity with regard to drugs such as Adriamycin, Epodyl, Mitomycin-c and Thiotepa in treating superficial bladder cancer (Parris C. et al. 1987) and increasing the antitumor efficiency of hyperthermia in treating B16 melanoma cells in BALB/C mice (Yaoqin Y. et al. 1996). It has also been shown that T80 decreases drug resistance in Actinomycin-D and Daunomycin resistant chinese Hamster cells (Riehm H. and Biedler J. 1972) and 
Daunorubicin resistant Ehrlich ascites cells (Sehested M. et al. 1989). In all of these cases, T80 acted as a solubilizing agent to the plasma membrane of cells (Jones M. 1999) as well as in increasing membrane fluidity (Basrur V. et al. 1983; Chitnis M. et al. 1984; Tsujino I. et al. 1999; Coors E. et al. 2005). This increase in the membrane fluidity could have significantly enhance the poration (nano) effect of nsPEFs where stable poration effect is seen for minutes (Bowman A. et al. 2010). Better formulation of possible effects of increased membrane fluidity in nsPEF according to reported effects in microsecond pulses in necessary (Kandušer M. et al. 2006), Another possible mechanism could be pore induced changes in the osmotic pressure of the cells leading to a increase in the size of cells (Fig.4) by the presence of T80. This effect, however, appeared to be cell type specific, with Jurakt cells showing significant swelling. Jurkat cells are non-adherant and have reduced supporting cytoskeletal networks comapred to adherent cells. The effects of nSPEF induced cytoskeleton damage (Stacey et al 2011) along with T80 induced membrane fluidity may disrupt electrolyte balance, followed by water uptake and thus making Jurkat cells more susceptible to osmotic swelling.

Even though Trypan Blue assay and WST-1 cell viability assay results showed significant killing effect of nsPEFs in presence of $0.18 \% \mathrm{~T} 80$, the difference in the cell viability results of these assays is primarily dependent on cell component involved with the assay. The Trypan Blue exclusion assay is based on the uptake of the dye by dead cells only. Whereas, the WST-1 cell viability assay is based on the cleavage of WST-1 into a soluble formazan dye by mitochondrial dehydrogenase of metabolically active and live cells to measure the number of metabolically live cells. Tween $80(0.5 \%$, Wallace et al 1968) has been implicated in mitochondrial and membrane biogenesis in yeast, a 
factor that was not investigated in these studies, but which may influence WST assay outcomes. Plasma membrane is significantly affected after exposure to nsPEFs (André F. M. et al. 2010) measured by propidium iodide uptake across the cell membrane. Trypan Blue assay is an assay which is based on the integrity of plasma membrane. Therefore, with viability assays that may be compromised by Tween 80 (WST), or

membrane permeability (Trypan Blue exclusion), the results of WST-1 cell viability assay and Trypan Blue assay may be expected to differ from each other.

\section{Conclusion:}

Based on our results, it can be concluded that T80 plays a major role in enhancing the killing effect of nsPEFs which could be due to the adjuvant effect on PANC1 and Jurkat cells. We believe that Tween 80 may increase the cell membrane permeability/fluidity leading to increased fragility of the cell membrane. Further investigation is necessary to understand the mechanism of T80 as an adjuvant in enhancing the killing effect of nsPEFs. We also conclude that cell viability depends upon the protocol employed and maybe indicative of different biological mechanism that respective cell viability assays measure as means of cell viability.

\section{Acknowledgement:}

We acknowledge the support of the Breeden Adams Foundation. The study sponsors had no role in study design, data collection, and data analysis; compiling the manuscript or in the decision to submit the short communication for publication. 


\section{References:}

André F M, Rassokhin M A, Bowman A M, et al. (2010). Gadolinium blocks membrane permeabilization induced by nanosecond electric pulses and reduces cell death, Bioelectrochemistry 79: 95-100.

Basrur V, Chitnis M, Menon R (1983). Differential Agglutination of P388 AdriamycinSensitive and P388 Adriamycin-Resistant Leukemia Cells, Oncology 40: 202204.

Beebe S, White J, Blackmore P, et al. (2003). Diverse effects of nanosecond pulsed electric fields on cells and tissues, DNA and cell biology 22: 785-796.

Beebe S J, Fox P M, Rec L J, et al. (2003). Nanosecond, high-intensity pulsed electric fields induce apoptosis in human cells, The FASEB journal 17: 1493-1495.

Bowman A, Nesin O, Pakhomova O, et al. (2010). Analysis of Plasma Membrane Integrity by Fluorescent Detection of Tl+ Uptake, Journal of Membrane Biology 236: 15-26.

Chen X, James Swanson R, Kolb J, et al. (2009). Histopathology of normal skin and melanomas after nanosecond pulsed electric field treatment, Melanoma Research 19: 361-371.

Chitnis M, Menon R, Gude R (1984). Effect of Tween 80 on adriamycin cytotoxicity in murine P388 leukemia, Tumori 70: 313.

Coors E, Seybold H, Merk H, et al. (2005). Polysorbate 80 in medical products and nonimmunologic anaphylactoid reactions, Annals of Allergy, Asthma \& Immunology 95: 593-599.

Jones M (1999). Surfactants in membrane solubilisation, International journal of pharmaceutics 177: 137-159.

Kandušer M, Šentjurc M, Miklav I D (2006). Cell membrane fluidity related to electroporation and resealing, European Biophysics Journal 35: 196-204.

Kolb J, Kono S, Schoenbach K (2006). Nanosecond pulsed electric field generators for the study of subcellular effects, Bioelectromagnetics 27: 172-187.

Nuccitelli R, Pliquett U, Chen X, et al. (2006). Nanosecond pulsed electric fields cause melanomas to self-destruct, Biochemical and biophysical research communications 343: 351-360.

Pakhomov A, Kolb J, White J, et al. (2007). Long-lasting plasma membrane permeabilization in mammalian cells by nanosecond pulsed electric field (nsPEF), Bioelectromagnetics 28: 655-663.

Parris C, Masters J, Walker M, et al. (1987). Intravesical chemotherapy: combination with Tween 80 increases cytotoxicity in vitro, Urological Research 15: 17-20.

Peskin A, Winterbourn C (2000). A microtiter plate assay for superoxide dismutase using a water-soluble tetrazolium salt (WST-1), Clinica Chimica Acta 293: 157166.

Riehm H, Biedler J (1972). Potentiation of drug effect by Tween 80 in Chinese hamster cells resistant to actinomycin D and daunomycin, Cancer Research 32: 1195.

Sabuncu A, Kalluri B, Qian S, et al. (2010). Dispersion state and toxicity of mwCNTs in cell culture medium with different T80 concentrations, Colloids and Surfaces B: Biointerfaces 78: 36-43. 
Schwan H, Chiabrera C, Nicolini C (1985). Interactions between electromagnetic fields and cells, Plenum Press, New York, pp 173-191:

Sehested M, Jensen P, Skovsgaard T, et al. (1989). Inhibition of vincristine binding to plasma membrane vesicles from daunorubicin-resistant Ehrlich ascites cells by multidrug resistance modulators, British journal of cancer 60: 809.

Stacey M, Fox P, Buescher S, Kolb J. (2011) Nanosecond pulsed electric field induced cytoskeleton, nuclear membrane and telomere damage adversely impact cell survival. Bioelectrochemistry. 82 131-134

Tennant J (1964). Evaluation of the trypan blue technique for determination of cell viability, Transplantation 2: 685.

Tsujino I, Yamazaki T, Masutani M, et al. (1999). Effect of Tween-80 on cell killing by etoposide in human lung adenocarcinoma cells, Cancer chemotherapy and pharmacology 43: 29-34.

Vernier P, Sun Y, Marcu L, et al. (2003). Calcium bursts induced by nanosecond electric pulses, Biochemical and biophysical research communications 310: 286-295.

Wallace PG, Huang M, Linnane AW. (1968). The Biogenesis of Mitochondria: II. The Influence of Medium Composition on the Cytology of Anaerobically Grown

Saccharomyces cerevisiae J.Cell Biol. vol. 37; 207-220

Yaoqin Y, Huchuan Y, Huihong T, et al. (1996). The enhancement of tween-80 on the antitumor effect of the hyperthermia $41^{\circ} \mathrm{C}$ in tumor-bearing mice, Chinese Journal of Cancer Research 8: 168-173.

Zheng Z, Obbard J (2002). Evaluation of an elevated non-ionic surfactant critical micelle concentration in a soil/aqueous system, Water research 36: 2667-2672. 